\title{
FLAVONOIDES E ANTOCIANINAS EM MYRCIARIA CAULIFLORA (JABUTICABA) VISANDO À APLICABILIDADE COSMÉTICA
}

\section{FLAVONOIDS AND ANTHOCYANINS IN MYRCIARIA CAULIFLORA (JABOTICABA) AIMING TO COSMETIC APPLICABILITY}

\author{
Nicole de Almeida Nunes MEIRA ${ }^{1^{*}}$; Neila de Paula PEREIRA ${ }^{1}$; Leonardo Fonseca \\ MACIEL $^{2}$; Douglas Dourado OLIVEIRA ${ }^{1}$; Ítalo Santos do NASCIMENTO'; Rousilândia \\ de Araújo SILVA ${ }^{1}$
}

1 - Laboratório de Pesquisa em Medicamentos e Cosméticos (LAPEMEC), Faculdade de Farmácia, Universidade Federal da Bahia.

2 - Laboratorio de Pesquisa e Análise de Alimentos e Contaminantes (LAPAAC), Universidade Federal da Bahia. *Autor para correspondência: nicole.nunes@gmail.com / nicole.nunes@ufba.br

\section{RESUMO:}

A jabuticaba se caracteriza por ser um fruto apreciado pelos brasileiros e muito usado na medicina popular. As cascas e sementes podem ser usados como fontes alternativas de micronutrientes e como ativos em indústrias de cosméticos. Este estudo tem como objetivo correlacionar a jabuticaba como potencial ativo para cosméticos sustentáveis. A revisão de literatura foi realizada consultando bases científicas, capítulos de livros temáticos, monografias de especialização, dissertações, teses e legislações no âmbito nacional e internacional. Também foi realizada uma prospecção patentária preliminar da relação entre jabuticaba e cosméticos. As antocianinas fazem parte do grupo dos flavonoides e estão presentes predominantemente em flores e frutos. Sua ação como antioxidante endógeno é considerada sua propriedade mais importante. Nas última décadas houve um aumento e envelhecimento da população mundial que juntamente com a busca por uma aparência mais jovem, tem despertado o interesse por estudos relacionados às propriedades antienvelhecimento das substâncias ativas. A aplicação de cosméticos tópicos com ativos antioxidantes reduz os danos oxidativos induzidos pela radiação UV e constituem uma boa alternativa na proteção da pele contra o fotoenvelhecimento. As cascas da jabuticaba possuem maior concentração de compostos fenólicos, maior atividade antioxidante e teor de antocianinas comparada com as outras frações. Apesar de todas as evidências promissoras que esta fruta e suas frações apresentam em relação à indústria cosmética, é necessário realizar mais pesquisas que identifiquem e afirmem com fontes científicas o seu potencial para ser utilizado como ativo cosmético.

Palavras-Chaves: Jabuticaba; Cosméticos; Flavonoides; Antocianinas; Antioxidante.

\section{ABSTRACT:}

The jaboticaba is very appreciated by the brazilians and is commonly used in the popular medicine. The peels and seeds can be used as alternative sources of micronutrients and as "active" in cosmetics. The aim of this study is correlate the jaboticaba and its potential as an active for sustainable cosmetics. The bibliographic research was made consulting scientific backgrounds, thematic books, monographs, essays, thesis and legislations in the international and national scope. In addition, a preliminary patent prospection about the 
relationship between jaboticaba and the cosmetics was made. The anthocyanin are part of the flavonoids. Its endogenous antioxidant action is considered as its most important property. During the last few decades, there was a rise and aging of the world population, which along with desire of maintaining a "young look", has awakening the interest in the for studies related to "anti-aging" of the actives substances. The topic cosmetics applications with antioxidants actives reduce the oxidative damages induced by UV radiation, and are a good alternative for the skin protection against "photo aging". Jaboticaba's peels have higher concentrations of phenolic compounds, of antioxidant activities and anthocyanin content, when compared to other fraction. Despite all the promising evidences that this fruit and its fractions present in relation with the cosmetic, it is necessary to do more researches that will help identify and confirm with scientific sources, its potential to be used as an active cosmetic.

Keywords: Jaboticaba; Cosmetic; Flavonoids; Anthocyanin; Antioxidant.

\section{INTRODUÇÃO}

De acordo com pesquisas realizadas em 2011 pelo Ministério do Meio Ambiente brasileiro, o Brasil é considerado o país da megadiversidade, com 15 a $20 \%$ das espécies do planeta. A composição total da biodiversidade brasileira não é conhecida e talvez nunca venha a ser, tal a sua magnitude e complexidade. Na lista da flora do Brasil, publicada em 2010, estão catalogadas 40.989 espécies (FORZZA, et al., 2010).

O estudo das plantas nativas contribui para uma melhor investigação dessas espécies, o que pode motivar novas atividades econômicas no país, e que muitas vezes são negligencias devido principalmente a falta de conhecimento (LIMA et al., 2010). A procura por um uso mais racional dos resíduos dos processos industriais de comidas e das partes não comestíveis das porções das frutas, pode acarretar em grandes benefícios para o meio ambiente, assim como para a humanidade, que reduzirá o desperdício de alimentos e contribuirá para o desenvolvimento de ações economicamente impactantes (LIMA, 2009; CRUZ, 2014).

Dentre os diversos tipos de frutas nativas e exóticas que existem no Brasil, podese destacar aquelas que fazem parte da família Myrtaceae. Essa família possui uma grande importância ecológica devido as suas características apícolas e por ter ampla ocorrência de espécies comestíveis e de frutas que são largamente utilizadas na medicina tradicional (PLAZA et al., 2007; ALMEIDA, 2011).

Entre as espécies frutíferas dessa família, pode-se destacar a jabuticabeira (Myrciaria cauliflora (DC) Berg), árvore de ocorrência espontânea em grande parte do Brasil, principalmente nos estados de Minas Gerais, Espírito Santo, Rio de Janeiro e Paraná (PEREIRA, 2003; BRUNINI et al., 2004). Embora popular em todo o Brasil, a jabuticaba não chega a ter valor comercial muito alto, por ser muito perecível. Apesar de ser grande a 
produção de um único pé e de ser considerada apropriada tanto para consumo in natura como para a indústria, depois de colhida, a fruta tem uma vida útil de até três dias, o que prejudica sua comercialização (BRUNINI et al., 2004; LIMA, 2009; CRUZ, 2014).

A jabuticaba se caracteriza por ser um fruto globoso, com casca avermelhada próxima ao negro, de polpa mucilaginosa, agridoce, altamente saborosa, e pode apresentar de uma a quatro sementes (REYNERTSON et al., 2006; LIMA et al., 2011; BORGES, 2014; LAGE, 2014; ANDRADE et al., 2015). Este fruto apresenta grande potencial de comercialização por ser muito apreciado não só para o consumo in natura como também para a fabricação de xaropes, geléias, bebidas fermentadas, licores caseiros, vinagres e sorvetes (PEREIRA, 2003; JESUS, 2004; CRUZ, 2014). A jabuticabeira tem despertado grande interesse entre os produtores rurais devido a sua alta produtividade, rusticidade e aproveitamento dos seus frutos em diversas formas (BRUNINI et al., 2004; BORGES, 2014; CRUZ, 2014).

Além do consumo das frutas, também atribui-se diversas atividades biológicas as jabuticabas que lhe conferem uso na medicina popular, alguns deles representados na tabela 1. As folhas e cascas de Myrciaria cauliflora são consideradas adstringentes e o seu decocto é empregado popularmente em diarréia e irritações de pele (SOUZA, 2007; GIRALDI, HANAZAKI, 2010; LEQUISTE et al., 2012; BORGES, 2014; ANDRADE, 2015). Pesquisadores investigaram a atividade antimicrobiana do extrato das folhas e do caule de M. cauliflora contra bactérias que formam o biofilme dental (Streptococcus mitis, Streptococcus mutans, Streptococcus sanguinis, Streptococcus oralis, Streptococcus salivarius e Lactobacillus casei) e os resultados encontrados foram positivos. (CARVALHO et al., 2009; MACEDO-COSTA, 2009; BORGES, 2014).

Um aumento no consumo das frutas leva também a um aumento nos resíduos, como as cascas e sementes que são subutilizadas ou não são utilizadas de forma alguma, em todos os pontos de comercialização até o consumo final, incluindo agricultores, indústrias e consumidores. Uma parte desse resíduo usualmente é utilizada in natura como alimentação animal, no entanto, a maior parte é descartada ou usada em compostagem (LIMA, 2009). Comumente as sementes são utilizadas apenas para a formação de mudas. Esses subprodutos podem ser usados como fontes alternativas de micronutrientes em indústrias de medicamentos e de cosméticos, que além de contribuir para diminuir o desperdício, reduz o impacto ambiental e agrega valor aos subprodutos (LIMA et al., 2011; BORGES, 2014; ZAGO, 2014).

As frutas tropicais são conhecidas por serem ricas fontes de compostos relacionados 
com a prevenção de doenças e prolongamento da vida. Os falvonoides, se destacam por serem antioxidantes naturais encontrados em frutas tropicais de coloração vermelha e roxa, capazes de inibir o processo de oxidação dos radicais livres, doando átomos de hidrogênio de maneira a inibir a reação de cadeia desses radicais (BIANCHI, ANTUNES, 1999; MAIA, 2013).

TABELA 1 - RELAÇÃO DO USO DA JABUTICABA NA MEDICINA POPULAR

\begin{tabular}{cc}
\hline Uso popular & Referencia \\
\hline Anti-inflamatória & Reynertson et al. (2006) \\
Transtornos respiratórios & Souza (2007) \\
Irritação da pele & Souza (2007) \\
Transtornos gastrointestinais & Giraldi (2010) \\
Hipoglicemia & Lenquiste et al. (2012) \\
Transtornos cardiovasculares & Andrade et al. (2015) \\
\hline
\end{tabular}

As substâncias antioxidantes naturais, como os falvonoides, são objetos de estudo da Cosmetologia, que pode ser definida como a ciência e a arte envolvidas na manutenção e melhoria dos caracteres celulares da pele. Portanto, pesquisadores da área das Ciências Cosméticas se preocupam constantemente com a prevenção e atenuação do envelhecimento cutâneo através de substâncias eficazes que possam desempenhar esses papéis (MAGALHÃES, 2000; SCOTTI et al., 2007).

Esta revisão bibliográfica tem como objetivo correlacionar a jabuticaba como potencial ativo para cosméticos sustentáveis. Ademais, vislumbra-se subsidiar a Pesquisa Desenvolvimento e Inovação (PD\&I) capaz de favorecer uma cosmetologia ecologicamente correta.

\section{METODOLOGIA}

O método de pesquisa adotado neste estudo baseou-se em uma revisão bibliográfica especializada realizado no período de janeiro e julho de 2016 . As buscas foram 
feitas mediante consultas nas bases científicas: Web of Science, MEDLINE®, BIREME, Science Direct e SciELO (Scientific Electronic Library Online). Além de capítulos de livros temáticos, monografias de especialização, dissertações, teses, legislações no âmbito nacional e internacional. Utilizou-se unitermos em português e/ou inglês de forma associada: jabuticaba (jaboticaba); cosméticos (cosmetics); falvonoides (flavonoids); antocianinas (anthocyanins); envelhecimento cutâneo (skin aging). Os materiais selecionados foram publicados entre os anos de 1999 a 2015.

Os critérios de inclusão compreenderam a disponibilidade eletrônica e a abordagem direta do tema, de forma a excluir aqueles não relacionados e as duplicidades encontradas. Assim sendo, utilizou-se um total de 57 materiais bibliográficos.

Também foi realizada uma prospecção patentária da relação entre jabuticaba e cosméticos, combinando-se os descritores: jabuticaba (jaboticaba), Myrciaria cauliflora e cosmético (cosmetic). Os bancos de patentes pesquisados foram o nacional INPI (Instituto Nacional de Propriedade Intelectual), e os internacionais, EPO (European Patent Office), USPTO (United States Patent and Trademark Office) e WIPO (World Intellectual Property Organization).

\section{REVISÃO BIBLIOGRÁFICA}

\subsection{Flavonoides: antioxidantes naturais}

Os compostos fenólicos se caracterizam por possuírem anel aromático com um ou mais substituintes hidroxílicos, incluindo seus grupos funcionais. O potencial antioxidante dos polifenóis em termos de atividade química ocorrem por meio da do- ação de elétrons ou átomos de hidrogênio aos radicais livres, ou como quelantes de metais, inibindo a formação de radicais livres catalisados por metais de transição (ANGELO, JORGE, 2007; SILVA, 2011; DELMONDES et al., 2013. LAGE, 2014).

Os falvonoides constituem um dos principais grupos de compostos fenólicos e estão presentes na maioria dos tecidos vegetais. Constituem um grupo de pigmentos vegetais amplamente distribuídos na natureza que dão cor a folhas, flores e fru- tos e desempenham funções de defesa, protegendo contra irradiação UV dentre outras (MARTÍNEZ-FLOREZ et al., 2002; LIMA, 2009; CIPRIANO, 2011; LAGE, 2014).

São subclassificados em seis subgrupos principais: flavonas, flavonóis, catequinas ou flavanóis, flavanonas, antocianinas e isoflavonas (VOLP et al., 2008; LIMA, 2009; SILVA, 
2011; DELMONDES, 2013). A estrutura básica desses compostos consiste em quinze carbonos repartidos em dois anéis aromáticos unidos via carbono heterociclíco de pirano, dispostos na configuração C6-C3-C6 chamada difenilpropano (BEHLING et al., 2004; SILVA, 2011; LAGE, 2014).

As antocianinas (das palavras gregas anthos, flor e kianos, azul) fazem parte do grupo dos falvonoides e são glicosídios de antocianidinas. Sua estrutura básica é o cátion flavílium (cátion 2-fenilbenzopirílio), que consiste de dois anéis aromáticos unidos por uma unidade de três carbonos, condensada por um oxigênio (CONSTANT, 2003; CIPRIANO, 2011; SILVA, 2011). Caracterizam-se por serem pigmentos que estão presentes predominantemente em flores e frutos e dão colorações que variam entre azul, vermelho e violeta. Os principais papéis biológicos das antocianinas incluem atração de insetos, fotoproteção, modulação da fotoinibição, potencialização da fotossíntese, além de atuarem como antioxidantes endógenos, considerada sua propriedade mais importante (MALACRIDA, MOTA, 2006; LIMA, 2009; SILVA, 2011).

$\mathrm{O} \mathrm{pH}$ é certamente o fator mais importante no que diz respeito à estabilidade $\mathrm{e}$ coloração das antocianinas. Em meio ácido, a $25{ }^{\circ} \mathrm{C}$, quatro estruturas coexistem em equilíbrio: o cátion flavilium, a base quinoidal, a pseudo base ou carbinol e a chalcona. As estruturas responsáveis pela coloração das antocianinas são o cátion flavilium e a base quinoidal, sendo o carbinol e a chalcona incolores. Soluções de antocianina apresentam uma coloração vermelha mais intensa em pH abaixo de 3,0. Aumentando para a faixa de 4,0 a 5,0 essa coloração tende a desaparecer (CONS- TANT, 2003; MALACRIDA, MOTA, 2006; SILVA, 2011).

\subsection{Antioxidantes e cosmetologia}

De acordo com dados do censo demográfico de 2010 do Instituto Brasileiro de Geografia e Ciências (IBGE), nas últimas décadas tem ocorrido um aumento da população mundial assim como o seu envelhecimento. Alinhando esses quesitos com a crescente tendência de busca por uma aparência mais jovem, cada vez mais os pesquisadores vêm se interessando pelos estudos relacionados às propriedades antienvelhecimento das substâncias ativas (BAIN \& COMPANY, 2014).

Uma das maiores causas do envelhecimento cutâneo é a desorganização do mecanismo de defesa antioxidante, provocando doenças na pele, que são resultados das condições causadas por esse desequilíbrio e que são consequências de danos a estruturas 
nela presentes, como lipídios, proteínas e DNA (MAGALHÃES, 2000; HIRATA, 2004).

Os raios solares contêm as radiações ultravioletas, que são extremamente energéticas, e comumente divididas em A (320-400nm), B (280-320nm) e C (100-290nm) (SCOTTI, et al., 2007). A radiação UVC, possui energia elevada e menor comprimento de onda. É altamente lesiva ao homem, com efeitos carcinogênicos e mutagênicos. No entanto, é absorvida em sua maioria pela camada de ozônio. As radiações UVA, menos energéticas, causam o bronzeamento imediato e de curta duração, além de penetrarem mais profundamente na pele atingindo a derme. São responsáveis pelo envelhecimento cutâneo precoce. Já a UVB, causa danos agudos, como queimaduras, sendo eritematógena, promovendo o bronzeamento tardio e de longa duração (SOUZA, et al., 2005; ARAÚJO, SOUZA, 2008).

Tanto UVA quanto UVB estimulam a formação de radicais livres, contudo, ainda não se tem certeza qual das duas provoca mais o aparecimento destas moléculas. $\mathrm{O}$ que se tem conhecimento é que as duas juntas são mais danosas que cada uma isoladamente e que o estresse oxidativo provocado por elas nas camadas da pele depende da penetração dos raios (SCOTTI, et al., 2007).

A aplicação tópica de antioxidantes reduz os danos oxidativos induzidos pela radiação UV, portanto, cosméticos de uso tópico contendo ativos antioxidantes em sua formulação, constituem uma boa alternativa na proteção da pele contra 0 foto envelhecimento (GUARATINI, et al., SCOTTI, et al., 2007; STEINER, 2008; FRIES, FRASSON , 2010).

Antioxidante é qualquer substância que, quando presente em baixa concentração comparada à do substrato oxidável, regenera o substrato ou previne significativamente a oxidação do mesmo (BARREIROS et al., 2006; TIVERON, 2010; PAIM, 2011; DELMONDES,2013). O mecanismo de ação dos antioxidantes permite classificá-los como: antioxidantes de prevenção, que impedem a formação de radicais livres; varredores, que impedem o ataque de radicais livres às células; de reparo, que favorecem a remoção de danos à molécula de DNA e a reconstituição das membranas celulares danificadas (KOURY, DONANGELO, 2003; STEINER, 2008; TELESI, MACHADO, 2008; DENADI, 2013).

De maneira natural, o organismo possui substâncias cujo objetivo é estabelecer um equilíbrio harmônico entra a presença das moléculas oxidantes, as antioxidantes e a pele. Por sua área extensa e sua função protetora do organismo ao meio, a pele fica muito exposta ao ataque dos radicais livres, portanto, a defesa antioxidante é constantemente 
requisitada (SCOTTI, et al., 2007). Desta maneira, a Cos- metologia se preocupa constantemente com a prevenção e atenuação do envelhec- imento cutâneo por meio de pesquisas de substâncias antioxidantes eficazes, que possam ser oferecidas em produtos cosméticos aos seus consumidores (MAGALHÃES, 2000; SCOTTI, et al., 2007; ANTERO, BORGES, 2011).

\subsection{Fenólicos totais, atividade antioxidante e antocianinas nas cascas da jabuticaba}

Estudos realizados para determinar os compostos fenólicos totais e a atividade antioxidante das frações da Jabuticaba constataram que a casca possui maior concentração de compostos fenólicos assim como a maior atividade antioxidante e a mais elevada capacidade de sequestrar o radical DPPH. A alta atividade antioxidante da casca da jabuticaba pode ser explicada pela elevada quantidade de antocianinas encontradas nessa parte do fruto (LIMA, 2009; REZENDE, 2010).

As cascas, quando comparadas com as polpas e sementes, apresentam o maior teor de antocianinas (LIMA, 2009). Comparando o teor de antocianinas presente nas cascas das jabuticabas com o teor da casca do açaí, do fruto da pitanga roxa e da uva, da casca do camu-camu fresco, das polpas de morango, amora, acerola, goiaba e repolho roxo e ainda das pétalas de hibisco e das cascas de beringela, constatou-se que casca da jabuticaba apresentou o maior teor de anto- cianinas. (BOBIO, et al., 2000; ZENNATA, et al., LIMA et al., 2005; KUSKOSKI et al., 2006; TEIXEIRA et al., 2008; LIMA, 2009; REZENDE, 2010; ARAÚJO, 2011). Os teores de cada espécie podem ser vistos na tabela 2 .

Nas cascas da jabuticaba foram encontradas duas antocianinas, que apesar de serem consideradas poucas em quantidades quando comparados a outros frutos, são consideradas de grande poder antioxidante, devido à estrutura que apresentam com duas hidroxilas (cianidina-3-glicosídeo) e três hidroxilas (delfinidina-3-glicosídeo) presentes no anel (LIMA, 2009; LEITE, 2010; MACHADO, 2013). 
TABELA 2 - Comparação das quantidades de antocianinas em espécies presentes no Brasil

\begin{tabular}{cccc}
\hline Espécie & Parte utilizada & $\begin{array}{c}\text { Quantidade } \\
(\mathbf{m g} / \mathbf{1 0 0 g})\end{array}$ & Referência \\
\hline Jabuticaba Sabará & Cáscara & 362,0 & Lima (2009) \\
Jabuticaba Paulista & Cáscara & 383,0 & Lima (2009) \\
Pitanga roxa & Fruto & 16,2 & Lima et al. (2005) \\
Camu-Camu & Cáscara & 54,0 & Zannata et al. (2005) \\
Uva & Fruto & 30,9 & Kuskoski et al. (2006) \\
Açaí & Cáscara & 263,0 & Bobio et al. (2000) \\
Morango & Pulpa & 21,69 & Teixeira et al. (2008) \\
Amora & Pulpa & 41,8 & Kuskoski et al. (2006) \\
Acerola & Pulpa & 16,0 & Kuskoski et al. (2006) \\
Goiaba & Pulpa & 2,7 & Kuskoski et al. (2006) \\
Hibisco & Pétalos & 250,97 & Teixeira et al. (2008) \\
Berinjela & Cáscara & 64,06 & Teixeira et al. (2008) \\
Couve rojo & Pulpa & 24,36 & Teixeira et al. (2008) \\
\hline
\end{tabular}

\section{PROSPECÇÃO PATENTÁRIA}

Os bancos de dados de patentes nacionais, INPI, e os internacionais, EPO, USPTO e WIPO, podem revelar informações como o quantitativo de patentes, a evolução anual de patentes, os países de depósito e os inventores depositantes. O quantitativo de patentes envolvendo jabuticaba e seu uso em cosméticos estão compilados na tabela 3.

TABELA 3 - Patentes da jabuticaba em cosméticos depositados em base de patentes.

\begin{tabular}{ccccc}
\hline EPO & USPTO & WIPO & INPI & Patentes total \\
\hline 1 & 0 & 1 & 0 & 2 \\
\hline
\end{tabular}

As duas patentes encontradas, na base EPO e WIPO, são as mesmas, intitulada "Cosmetic composition containing moisturizing plant extract" e foram depositadas como PTC (Tratado de Cooperação de Patentes), no qual paga-se para a mesma patente ficar protegida em mais de um território. 


\section{CONSIDERAÇÕES FINAIS}

Poucos estudos são encontrados na literatura em relação aos constituintes químicos da jabuticaba, principalmente em relação as suas frações e sua relação com cosméticos. Da mesma forma, a produção comercial, embora esteja em crescimento, ainda é pequena e limitada a determinadas regiões, sendo ainda considerada uma planta frutífera de pomares domésticos, de chácaras, sítios ou fazendas (SATO, CUNHA, 2007; LIMA, 2009; CRUZ, 2014).

Referente ao cenário patentário, em uma busca preliminar, observa-se um perfil semelhante ao da literatura científica, visto que nas bases de dados analisadas, encontrouse apenas uma patente, que foi depositada em duas bases distintas.

Dessa maneira, ampliar os conhecimentos acerca dos constituintes dessas frações leva a um maior aproveitamento da fruta, agregando-Ihe valor. $O$ estudo de frutas nativas ou típicas do Brasil, que ainda são pouco conhecidas e muito negligenciadas, representa uma contribuição relevante, podendo auxiliar na exploração de espécies nacionais que culminam no desenvolvimento de novas atividades econômicas, motivando grandes investimentos por partes das indústrias alimentícias, farmacêuticas e cosméticas, além de despertar 0 interesse de pesquisas inovadoras, principalmente de países mais desenvolvidos. É fundamental que o Brasil intensifique investimentos e implemente programas de pesquisa na busca de um melhor aproveitamento desse imenso patrimônio natural (LIMA, 2009; MINISTÉRIO DO MEIO AMBIENTE, 2011).

Apesar de todas as evidências promissoras que esta fruta e suas frações apresentam em relação à indústria cosmética, pouquíssimos estudos são encontra- dos na literatura que justifiquem o seu uso. Portanto, é nítida a necessidade de se realizarem mais pesquisas que identifiquem e afirmem com fontes científicas o seu potencial para ser utilizado como ativo cosmético.

\section{REFERÊNCIAS}

ANDRADE, D.M.L. et al. Vasorelaxant and Hypotensive Effects of Jaboticaba Fruit (Myrciaria Cauliflora) Extract in Rats. Evidence-Based Complementary and Alternative Medicine.v. 2015, 2015.

ANGELO, P.M.; JORGE, N. Compostos fenólicos em alimentos - Uma breve revisão. 
Revista do Instituto Adolfo Lutz. v. 66, p. 232-240, 2007.

ANTERO, R.V.P.; BORGES, E.C.L. Identificação de fitonutrientes antioxidantes em frações vegetais de maçã para o desenvolvimento de fitocosmético. Instituto Federal de Educação, Ciência e Tecnologia de Goiás - Campus Inhumas II SIMPOETS. Goiás, v. 2, n.1, p. 101$104,2011$.

ARAÚJO, C.R.R. Composição química, potencial antioxidante e hipolipidêmico da farinha da casca de Myrciaria cauliflora (jabuticaba). Tese (Mestrado em Química Orgânica)Universidade Federal dos Vales do Jequitinhonha e Mucuri, Di- amantina, 2011.

ARAÚJO, T.S. SOUZA, S.O. Protetores solares e os efeitos da radiação ultravioleta. Scientia Plena. v. 4, n.11, 2011.

BAIN \& COMPANY. Potencial de diversificação da indústria química brasileira - Relatório 04: Cosméticos e Higiene Pessoal. Rio de Janeiro, 2014. Disponível em: <http://www.bndes.gov.br/ SiteBNDES/export/sites/default/bndes_pt/Galerias/Arquivos/produtos/download/aep_fep/c hamada_publica_FEPprospec0311_Quimicos_Relat4_cosmesticos.pdf $>$. Acesso em: 13 jul. 2016.

BARREIROS, A. L. B. S. DAVID, J. M. ; DAVID, J. P. Estresse oxidativo: relação entre geração de espécies reativas e defesa do organismo. Química Nova. v. 29, n.1, p. 113-123, 2006.

BEHLING, E.B. et al. Flavonóide quercetina: aspectos gerais e ações biológicas. Alimentos e Nutrição Araraquara, v. 15, n. 3, p. 285-29, 2004.

BIANCHI, M. D. A. ANTUNES, L. M.G. Radicais livres e os principais antioxidantes da dieta. Artigo de revisão. Revista de Nutrição. v. 12, n. 2, p. 123-130, 1999.

BORGES, L.L. Bioprodutos padronizados em compostos fenólicos obtidos de resíduos agroindustriais das cascas dos frutos de Myrciaria cauliflora (Mart.)

O. Berg. Tese (Doutorado em Ciências Farmacêuticas) - Universidade de Brasília, Brasília, 2014. 
BOBBIO, F. O. et al. Identificação e quantificação das antocianinas do fruto do açaizeiro (Euterpe oleracea) Mart. Ciência e Tecnologia de Alimentos. v. 20, n. 3, p. 388-390, 2000. BRUNINI, M.A. et al. Influência de embalagens e temperatura no armazenamento de jabuticabas (Myrciaria jabuticaba (vell) berg) cv 'sabará'. Ciência e Tecnologia de Alimentos. v. 24, n. 3, p. 378-383, 2004.

CARVALHO, C.M. et al. Efeito antimicrobiano in vitro do extrato de jabuticaba [Myrciaria cauliflora (Mart.)O.Berg.] sobre Streptococcus da cavidade oral. Revista Brasileira de Plantas Medicinais. v.11, n.1, p 79-83, 2009.

CIPRIANO, P.A. Antocianinas de açaí (Euterpe oleracea mart.) e casca de jabuticaba (Myrciaria jaboticaba) na formulação de bebidas isotônicas. Dissertação (Mestrado em Ciências e Tecnologia de Alimentos) - Universidade Federal de Viçosa,Viçosa - MG, 2011.

CONSTANT, P.B.L. Extração, caracterização e aplicação de antocianinas de açaí (Euterpe oleraea, M.). Tese (Doutrorado em Ciências e Tecnologia de Alimentos) - Universidade Federal de Viçosa, Viçosa, 2003.

CRUZ, E. S. da. Caracterização de genótipos de jabuticabeira com base em descritores morfoagronômicos e moleculares. Dissertação (Mestrado em Recursos Genéticos Vegetais) - Cruz das Almas, BA, 2014.

DELMONDES, P.H. Substâncias antioxidantes presentes nos vegetais. Interdisciplinar: Revista Eletrônica da Univar. v.1, n. 09, p. 1- 5, 2013.

DENADI, A.S. Análise dos efeitos agudos do laser de baixa intensidade $(660 \mathrm{~nm})$ sobre os níveis de estresse oxidativo de ratos diabéticos com feridas na pele. Tese (Doutorado em em Saúde e Desenvolvimento) - Universidade Federal de Mato Grosso do Sul, Campo Grande, 2013.

DRAGANO, N. R. V. et al. Freeze-dried jaboticaba peel powder improves insulin sensitivity in high-fat-fed mice. British Journal of Nutrition. v. 110, n. 3, p. 447-455, 2013.

FORZZA, R. C. et al. Catálogo de plantas e fungos do Brasil. Instituto de Pesquisa Jardim 
botânico do Rio de Janeiro, 2010.

FRIES, T.A.; FRASSON, A.P. Z. Avaliação da atividade antioxidante de cosméticos antiidade. Revista Contexto \& Saúde. v.10, n. 19, p.17-23, 2010.

GIRALDI, M.; HANAZAKI, N. Use and traditional knowledge of medicinal plants at Sertão do Ribeirão, Florianópolis, Santa Catarina State, Brazil. Acta Botanica Brasilica. v. 24, n. 2, p. 395-406, 2010.

GUARATINI, T. et al. Antioxidantes na manutenção do equilíbrio redox cutâneo: uso e avaliação de sua eficácia. Química Nova. v. 30, n. 1, p. 206-213, 2007.

HIRATA, L.L. et al. Radicais Livres e o Envelhecimento Cutâneo. Acta Farmacéutica Bonaerense. v. 23, n. 3, p. 418-424, 2004.

INSTITUTO BRASILEIRO DE GEOGRAFIA E ESTATÍSTICA. Brasil em síntese. Disponível em: < http://brasilemsintese.ibge.gov.br/populacao/distribuicao-da-populacao-por-grandesgrupos-de-idade.html>. Acesso em: 18 maio 2016.

JESUS, N. Caracterização de quatro grupos de jabuticabeira, nas condições de Jaboticabal-SP. Revista Brasileira de Fruticultura. v. 26, n. 3, p. 482-485, 2004.

KOURY, J.C. DONANGELO, C.M. Zinco, estresse oxidativo e atividade física. Revista de Nutrição. v. 16, n. 4, p. 433-441, 2003.

KUSKOSKI, E.M. et al. Frutos tropicais silvestres e polpas de frutas congeladas: atividade antioxidante, polifenóis e antocianinas. Ciência Rural. v. 36, n. 4, p. 1283-1287, 2006.

LAGE, F.F. Casca de jabuticaba: inibição de enzimas digestivas, antioxidante, efeitos biológicos sobre o fígado e perfil lipídico. Tese (Doutorado em Agroquímica) - Universidade Federal de Lavras. Lavras - MG, 2014.

LEITE, A.V. Avaliação da composição e da capacidade antioxidante "in vivo" e "in vitro" de antocianinas da casca de jabuticaba (Myrciaria jaboticaba (Vell.) Berg) liofilizada em ratos 
Wistar. Tese (Mestrado em Alimentos e Nutrição) - Universidade Estadual de Campinas, Campinas, 2010.

LENQUISTE, S. A. ett al. Freeze-dried jaboticaba peel added to high-fat diet increases HDLcholesterol and improves insulin resistance in obese rats. Food Research International. v. 49, n. 1, p. 153-160, 2012.

LIMA, A. de J. B. et al. Sugars, organics acids, minerals and lipids in jabuticaba. Revista Brasileira de Fruticultura. v. 33, n. 2, p. 540-550, 2011.

LIMA, A. J. B. Caracterização e atividade antioxidante da jabuticaba, [Myrciaria cauliflora (Mart.) O. Berg]. Tese (Doutorado em Agroquímica)- Universidade Federal de Lavras, Lavras, 2009.

LIMA, V.L.A.G et al. Efeito da luz e da temperatura de congelamento sobre a estabilidade das antocianinas da pitanga roxa. Ciência e Tecnologia de Alimentos. v. 25, n. 1 p. 92-94, 2005.

LOPES, R.M. et al. Flavanoides. Biotecnologia Ciência \& Desenvolvimento. v. 3, n. 14, p. 18-22, 2000.

MACHADO, A.M.R. et al. Identificação e quantificação de antocianinas na casca de jabuticaba liofilizada. Communication to XVIII Encontro Nacional de Analistas de Alimentos - ENAAL IV Congresso Latino Americano de Analistas de Alimentos. São Paulo, São Paulo, 2013.

MACEDO-COSTA, M.R. et al. Atividade antimicrobiana e antiaderente de Mimosa tenuiflora (Willd) Poir e Myrciaria cauliflora Berg sobre bactérias do biofilme dental. Revista Brasileira de Plantas Medicinais. v. 11, n. 1, p. 79-83, 2009.

MAGALHÃES, J. O uso de cosméticos através dos tempos, envelhecimento cutâneo. Cosmetologia: com questões de avaliação. Rio de Janeiro, 2000.

MAIA, J.L. Desenvolvimento de microcápsulas contendo as antocianinas presente no corante 
do extrato do jambo por polimerização interfacial. Tese (Mestrado em Engenharia Química) - Universidade Federal do Rio Grande do Norte, Na- tal, 2013.

MALACRIDA, C.R.; MOTTA, S. Antocianinas em suco de uva: composição e estabilidade. Boletim do Centro de Pesquisa de Processamento de Alimentos. v. 24, n. 1, p. 59-82, 2006.

MARTÍNEZ-FLÓREZ et al. Los flavanoides: propriedades y acciones antioxidantes. Nutrición Hospitalaria. v. 17, n. 6, p. 271-278, 2002.

MINISTÉRIO DO MEIO AMBIENTE. Espécies Nativas da Flora Brasileira de Valor Econômico. Atual ou Potencial Plantas para o Futuro - Região Sul. Brasília, 2011.

PAIM, A.N. Utilização de gordura oxidada em dietas de frangos de corte. Dissertação (Mestre em Ciências Veterinárias)- Universidade Federal do Paraná. Curitiba, 2011.

PLAZA, C. V.; SILVA, D. H. S.; PAULETTI, P. M. Antioxidantes presentes em folhas e frutos de Eugenia jambolana Lam. (Myrtaceae). In: Reunião Anual da Sociedade Brasileira de Química. Águas de Lindóia. Anais. Águas de Lindóia: Sociedade Brasileira de Química, 2007.

REYNERTSON, K. A. et al. Bioactive Depsides and Anthocyanins from Jaboticaba (Myrciaria cauliflora). Journal of Natural Products. v. 69, n. 8, p. 1228-1230, 2006.

REZENDE, L.C. Avaliação da atividade antioxidante e composição química de seis frutas tropicais consumidas na Bahia. Tese (Doutorado em Química) - Universidade Federal da Bahia, Programa de Pós-Graduação em Química, Salvador, 2010.

SATO, A.C.K.; CUNHA, R.L. Influência da temperatura no comportamento reológico da polpa de jabuticaba. Ciência e Tecnologia de Alimentos. v. 27, n. 4, p. 890-896, 2007.

SCOTTI, L. et al. Modelagem molecular aplicada ao desenvolvimento de moléculas com atividade antioxidante visando ao uso cosmético. Revista Brasileira de Ciências Farmacêuticas. v. 43, n. 2, p. 153-166, 2007. 
SILVA, P.I. Otimização da extração e microencapsulamento de polifenóis e an- tocianinas de jabuticaba (Myrciaria Jaboticaba). Tese (Doutorado em Ciência e Tecnologia de Alimentos) - Universidade Federal de Viçosa, Viçosa-MG, 2011.

SOUZA, T.M. et al. Avaliação da atividade fotoprotetora de Achillea millefolium L. (Asteraceae). Revista Brasileira de Farmacognosia. v. 15, n. 1, p. 36-38, 2005.

SOUZA, T. M. Estudo farmacognóstico e avaliação da atividade antimicrobiana e citotóxica de preparações cosméticas contendo o extrato de folhas de Myr- ciaria cauliflora O. Berg. (Myrtaceae) e de casca de Stryphnodendron adstrin- gens (Mart.) Coville (LeguminosaeMimosoidae). Dissertação (Mestrado em Ciências Farmacêuticas) - Universidade Estadual Paulista, Araraquara, 2007.

STEINER, D. Antioxidantes em cosméticos. Cosmetics \& Toiletries, São Paulo, v. 20, n. 4, p. 36, 2008.

TALESI, M. MACHADO, F.A. A influência do exercício físico e dos sistemas antioxidantes na formação de radicais livres no organismo humano. Revista de Saúde e Biologia. v. 3, n. 1 p. $40-49,2008$.

TEIXEIRA, L.N. et al. Comparação de métodos para quantificação de antocianinas. Revista Ceres. v. 55, p. 297-304, 2008.

TIVERON, A.P. Atividade antioxidante e composição fenólica de legumes e verduras consumidos no Brasil. Dissertação (Mestrado em Ciência e Tecnologia de Alimentos) Universidade de São Paulo, Piracicaba, 2010.

ZAGO, M.F.C. Aproveitamento de resíduo agroindustrial de jabuticaba no desenvolvimento de formulação de cookie para a alimentação escolar. Dissertação (Mestrado em Ciência e Tecnologia de Alimentos) - Universidade Federal de Goiás, Goiânia, 2014.

ZANATTA, C. F. et al. Determination of anthocyanins from Camu-camu (Myrciaria dúbia) by HPLC-PDA, HPLC-MS, and NMR. Journal of Agricultural and Food Chemistry, Easton. v. 53, n. 24, p. 9531-9535, 2005. 